Article

\title{
Optimized Soft Lithography Method for Polymer Cholesteric Liquid Crystal Flakes Fabrication
}

\author{
Guanqing Zhou ${ }^{1,3,+}$, Sunqian Liu ${ }^{1,2,+}$, Wei Liu ${ }^{1,2}$, Dong Yuan ${ }^{1,2, *(1)}$ and Guofu Zhou ${ }^{1,2}$ \\ 1 Guangdong Provincial Key Laboratory of Optical Information Materials and Technology \& Institute of \\ Electronic Paper Displays, South China Academy of Advanced Optoelectronics, South China Normal \\ University, Guangzhou 510006, China \\ 2 SCNU-TUE Joint Lab of Device Integrated Responsive Materials (DIRM), National Center for International \\ Research on Green Optoelectronics, South China Normal University, No 378, West Waihuan Road, \\ Guangzhou Higher Education Mega Center, Guangzhou 510006, China \\ 3 Department of Physics and Astronomy and Collaborative Innovation Center of IFSA (CICFSA), \\ Shanghai Jiao Tong University, Shanghai 200240, China \\ * Correspondence: yuandong@scnu.edu.cn \\ + These authors contributed equally to this work and should be considered co-first authors.
}

Received: 27 May 2019; Accepted: 25 June 2019; Published: 1 July 2019

\begin{abstract}
Polymer cholesteric liquid crystal (PCLC) flakes are gaining increasing interest for a wide variety of applications because of their unique optical properties and capabilities. Soft lithography is the most effective way to fabricate regularly shaped PCLC flakes. However, it is not easy to peel the flakes from the mold without breaking them. In order to peel the PCLC flakes from the patterned polydimethylsiloxane (PDMS) mold in a convenient way, in this paper, a method of coating a layer of polyvinyl alcohol (PVA) on a PDMS mold was proposed. The influence of the thickness of the PVA layer on the shape of the PCLC flakes and the release time from the PDMS mold were investigated. The results show that the presence of the PVA layer can speed up the release of the PCLC flakes and help maintain the shape effectively. Notably, the utilization of a PVA layer makes the PDMS mold recyclable. The influence of PCLC flake shape was also studied. This work will promote the development of switchable PCLC flake-based technologies.
\end{abstract}

Keywords: soft lithography; polymer cholesteric liquid crystal; flakes; polyvinyl alcohol layer; release time

\section{Introduction}

Polymer cholesteric liquid crystals (PCLCs) exhibit unique wavelength and polarization selective reflection owing to their molecular structure which is ordered in layers and rotates around an axis normal to the layers [1]. Because the glass-transition temperatures are well above room temperature, PCLCs can be manufactured as stable films, flakes or particles [2-4].

Switchable PCLC flake and particle-based technologies are gaining increasing interest for a wide variety of applications because of their unique optical properties and capabilities. An obvious application for switchable flakes is reflective displays which require low power consumption and have a paper-like visual appearance due to the use of ambient light. The reflective display (e-paper) market is predicted to grow dramatically in the coming years as it moves into applications which are currently dominated by traditional paper. PCLC flake technology for reflective display uses flakes for reflection, which is similar to electrophoretic particles (EPD), but based on the unique reflective properties of PCLC flakes [1,2]. PCLC flakes can also be used in infrared reflective device. The alignment direction of PCLC flakes in the device is driven by the rotation of a host liquid crystal in an electric field, which realize the switch from the infrared reflection state to the transmission state [5]. 
To develop commercially switchable flake-based technologies, one of the key factors is the fabrication of PCLC flakes. Several methods have been proposed over the past decades [5-7]. PCLC flakes have been produced by freeze-fracturing a PCLC film to break it down into irregularly shaped flakes with a length in the range of tens to hundreds of microns [8]. Irregular PCLC flakes can also be fabricated by ultrasonic crushing [5]. However, these two methods are not that effective and consume additional energy. In order to obtain well-defined and shaped PCLC flakes, a soft lithography method using a patterned polydimethylsiloxane (PDMS) mold is commonly used. Trajkovska-Petkoska fabricated shaped PCLC flakes using soft lithography and found that the shaped flakes were smooth and uniform in size, shape and thickness [9]. Trajkovska-Petkoska also found that PCLC flakes exhibit unique optical properties, such as selective reflection and circular polarization of reflected light [10].

However, it is not easy to peel the PCLC flakes from the PMDS mold without breaking it during soft lithography fabrication. From Figure 1 we can see that the flakes might stick to the PDMS mold which will lead to problems upon release of the flakes. Moreover, in Trajkovska-Petkoska's work, they quenched the PDMS mold and could not peel the all flakes away, as some of the flakes adhered to the PDMS mold. Also quenching the PDMS mold may damage it.

To overcome the shortcoming of soft lithography, in this paper, a method of coating a polyvinyl alcohol (PVA) layer on the PDMS mold was proposed, which helps to release the PCLC flake. The influence of the thickness of the PVA layer on the shape of the PCLC flakes and the release time from the PDMS mold were investigated. The result shows that the PVA layer can speed up the release of PCLC flakes and effectively help to maintain the shape.
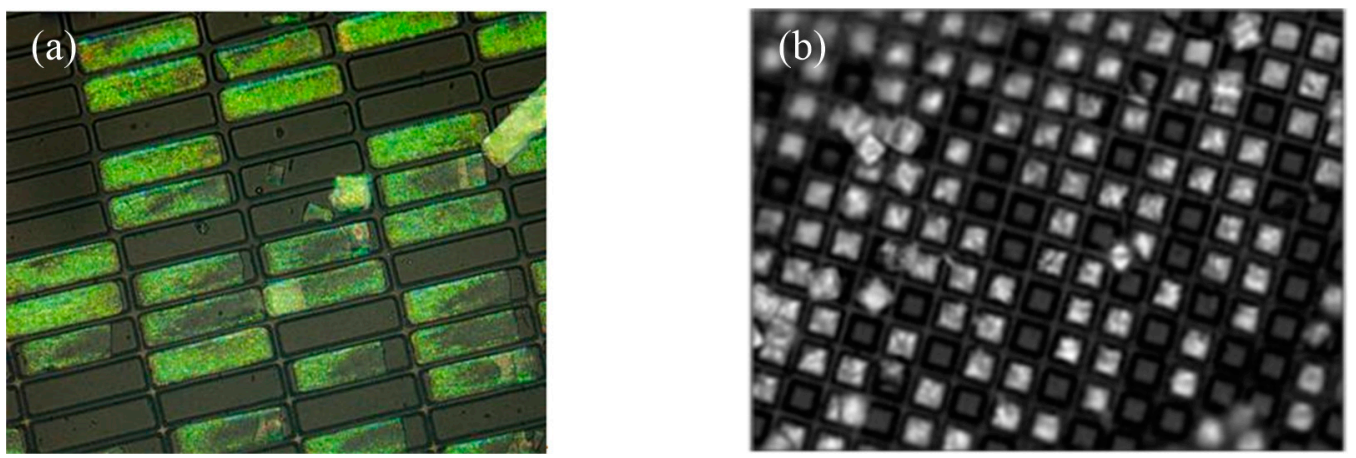

Figure 1. Flakes stick to the polydimethylsiloxane (PDMS) mold and cannot be peeled off: (a) Flakes fabricated by us without a polyvinyl alcohol (PVA) layer; (b) flakes in Trajkovska-Petkoska's paper [9], Copyright 2005, John Wiley and Sons.

\section{Materials and Methods}

\subsection{Materials and Preparation}

An overview of the materials used to fabricate the PCLC flakes is shown in Scheme 1. Monomers 1-4 were all obtained from Jiangsu Hecheng new materials Co. Ltd., Nanjing, China. Monomer 1 is a di-acrylate cross-linker. Monomers 2 and 3 are mono-acrylates. The phase transition temperature and mechanical strength of the mixed material change when the ratio between 1, 2 and 3 is varied. Monomer 4 is a chiral dopant which allows variation of the pitch lengths of PCLC by controlling its concentration. Photo-initiator 5 was obtained from BASF SE, Ludwigshafen, Germany, and was added to allow photo-initiation of the polymerization by exposure to UV light. Monomer 6 is a surfactant which induces planar alignment at the interface of the liquid crystal (LC) material and air. It was obtained from Shenzhen Regent Biochemistry Technology Co. Ltd., Shenzhen, China. Typically, the liquid crystal mixtures were fabricated from monomer 1 , monomer 2 , monomer 3 (the proportion of these three monomers was 2:3:4), monomer 4 whose concentration will be determined by the target reflection band (about 3-4 wt.\% in this paper), $2 \mathrm{wt} . \%$ of monomer 5, $0.5 \mathrm{wt} . \%$ of monomer 6 , and 200 ppm of polymerization inhibitor. 


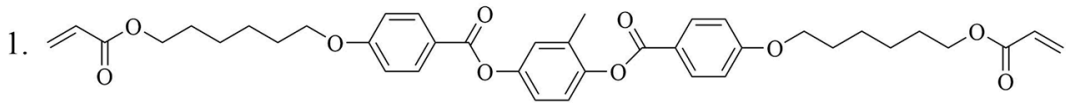

[3-methyl-4-[4-(6-prop-2-enoyloxyhexoxy)benzoyl]oxyphenyl] 4-(6-prop-2-enoyloxyhexoxy)benzoate

2 .

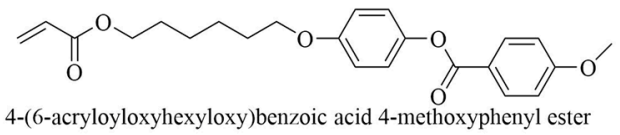

3.

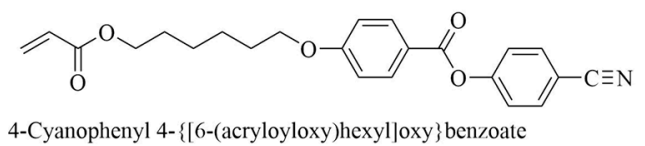

4.

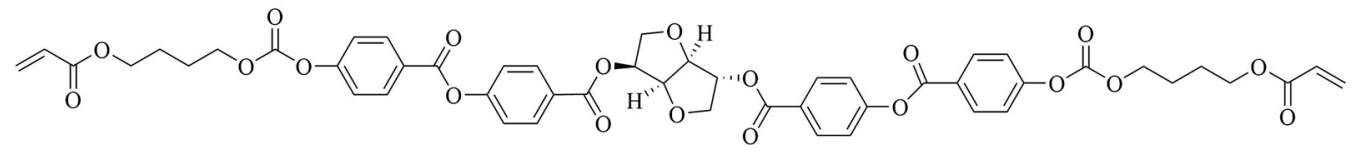

1,4:3,6-Dianhydro-D-glucitol bis[4-[[4-[[[4-[(1-oxo-2-propenyl)oxy]butoxy]carbonyl]oxy]benzoyl]oxy]benzoate]

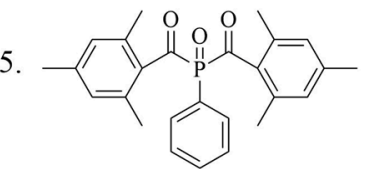

Phenylbis(2,4,6-trimethylbenzoyl)phosphine oxide

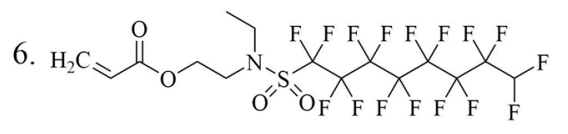

2-(N-Ethylperfluorooctanesulfonamido) ethyl methacrylate

Scheme 1. Chemical structure of the monomers used for the preparation of the polymer cholesteric liquid crystals flakes.

The monomers were mixed by dissolving them in dichloromethane, which was subsequently evaporated. A Differential Scanning Calorimeter (DSC) result shows that the mixture was in the cholesteric phase in the temperature range between $40{ }^{\circ} \mathrm{C}$ and $65^{\circ} \mathrm{C}$, as shown in Figure $\mathrm{S} 1$ in the Supplementary Material. At higher temperatures it became isotropic.

\subsection{PDMS Mold Manufacture}

Figure 2 shows the schematic diagram of the PDMS mold manufacturing process. In order to fabricate the well-defined PDMS mold, photo lithography was used to manufacture a patterned photoresist wafer. The detailed photo lithography process is as follows: A layer of photoresist (HN-008, Suntific Materials Co., Ldt., Weifang, China) was spin-coated over the glass substrate with $450 \mathrm{rpm}$ rotation speed for $30 \mathrm{~s}$. The photoresist was pre-baked at $110^{\circ} \mathrm{C}$ for $5 \mathrm{~min}$ to make the photoresist adhere to the surface of glass substrate, and the sample was cooled down to the room temperature afterward. Exposure with mask on the lithography machine (URE-2000/35, Institute of Optics and Electronics, Chinese Academy Science, Chengdu, China) for $10 \mathrm{~s}$, the intensity of UV light was $27 \mathrm{mw} / \mathrm{cm}^{2}$ and the wavelength was $365 \mathrm{~nm}$. During exposure, the exposed part of photoresist was cross-linked. The photoresist was baked again at $110^{\circ} \mathrm{C}$ for $5 \mathrm{~min}$ to ensure that the cross-linked part can stayed on the glass substrate after development. The sample was immersed in $0.4 \% \mathrm{KOH}$ for 3 min to develop the patterns; the unexposed photoresist dissolved in the develop solution patterns were formed. The height/depth of the patterns on the PDMS mold was controlled by the thickness of the photoresist. A PDMS mixture was poured over the photoresist wafer section, allowed to cure for $1 \mathrm{~h}$ at $60^{\circ} \mathrm{C}$, and then peeled off. The patterns on PDMS molds used in this paper had 4 different sizes: $30 \mu \mathrm{m} \times 30 \mu \mathrm{m}$, $20 \mu \mathrm{m} \times 60 \mu \mathrm{m}, 30 \mu \mathrm{m} \times 60 \mu \mathrm{m}$, and $60 \mu \mathrm{m} \times 60 \mu \mathrm{m}$. 
(a)

Pouring photoresis
over the mask
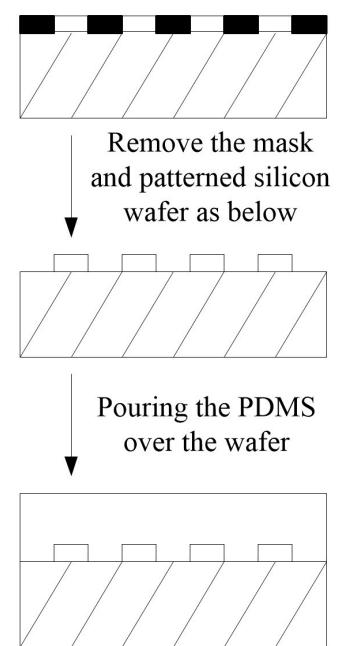

Curing and peeling

$\checkmark$ off the PDMS mold

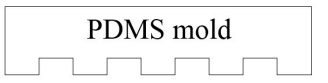

(b)

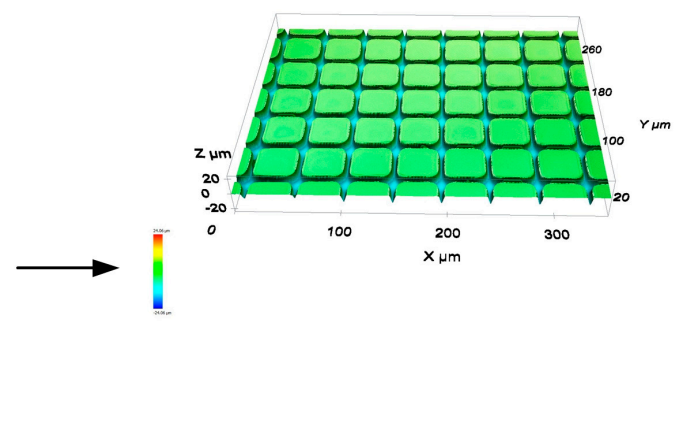

(c)

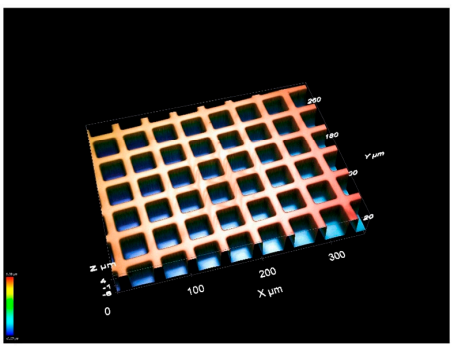

Figure 2. PDMS mold for soft lithograph: (a) A schematic illustration of the PDMS mold manufacturing process; (b) A patterned silicon wafer with a $10 \mu \mathrm{m}$ high square pattern; (c) A PDMS mold made using the patterned silicon wafer shown in $b$.

\subsection{The Manufacture of PCLC Flakes}

The method for manufacturing regularly shaped PCLC flakes is shown in Figure 3. The process flow is as follows: (a) The $5 \mathrm{wt} . \%$ PVA (MW:9000-10000, grade of hydrolysis: $80 \%$, Sigma-Aldrich, St. Louis, MO, USA) was dissolved into water and stirred for $5 \mathrm{~h}$ at $50{ }^{\circ} \mathrm{C}$, then the PVA solution was spin-coated onto the PDMS mold; (b) the PDMS mold was placed on a $60^{\circ} \mathrm{C}$ hot plate for $1 \mathrm{~h}$ to dry the PVA; (c) the PDMS mold was filled with LC mixture and blade coating was applied to the LC mixture; (d) a glass plate with a rubbed parallel alignment layer was put over the PDMS mold containing the LC mixture and the glass was pushed in order to squeeze out the redundant LC mixture and align the LC molecules with the rubbed parallel alignment layer on the glass; (e) the sample was slowly cooled down on the hot plate to $45^{\circ} \mathrm{C}$, then the upper glass plate was removed and "knife-coating" was applied to the sample with the same glass plate; the sharing force of knife-coating was used to enhance the alignment of LC molecules; (f) the LC mixture was polymerized using a UV light source under nitrogen atmosphere at $45^{\circ} \mathrm{C}$ for $5 \mathrm{~min}$; the intensity of the UV light source (OmniCure S1500, EXFO Photonic Solutions Inc., Mississauga, ON, Canada) we used was $200 \mathrm{mw} / \mathrm{cm}^{2}$. The wavelength of the UV light was $320-500 \mathrm{~nm}$; (g) post cure was achieved by heating at $125^{\circ} \mathrm{C}$ for $15 \mathrm{~min}$ and then slowly cooled down to room temperature on the hot plate; (h) the sample was immersed in water and the ultrasonic vibration was used to release the PCLC flakes from the molds. 


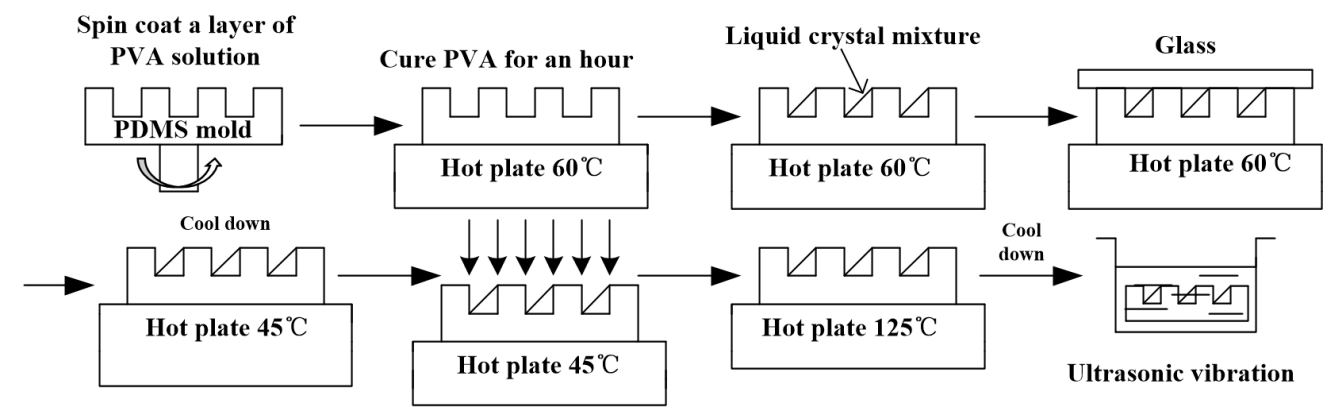

Figure 3. Fabrication process of polymer cholesteric liquid crystals (PCLC) flakes using soft lithography.

\subsection{Characterization and Measurements}

The phase transition temperatures of the mixtures were studied using Differential Scanning Calorimeter (DSC, METTLER DSC 1, Mettler Toledo, Inc., Zurich, Switzerland). The heating and cooling rates were both $2{ }^{\circ} \mathrm{C} / \mathrm{min}$. Optical images between crossed polarizers were taken using a polarized optical microscope (POM, Leica DM 2700 P, Leica Microsystems Ltd., Wetzlar, Germany). Transmittance spectra of the reflectors were measured using an Ocean Optics spectrometer 2000 PRO. The surface topography of PCLC flakes was measured using a 3D surface profilometer (Leica DCM8, Leica Microsystems Ltd., Wetzlar, Germany). In order to release PCLC flakes, we immersed the PDMS mold filled with flakes in water and shook it using the ultrasonic cleaner (SG3200HBT, Shanghai Guante ultrasonic instrument co., Ltd., Shanghai, China). The status of flake release was checked every minute. When there were no flakes left on the mold, the total ultrasonic time was recorded as the release time of the PCLC flakes.

\section{Results and Discussion}

\subsection{Characterization of Shaped Flakes}

The shape, alignment, and morphology of the PCLC flakes with $30 \mu \mathrm{m} \times 30 \mu \mathrm{m}$ size in the PDMS mold with $0.5 \mu \mathrm{m}$ PVA layer were studied, as shown in Figure 4. From the POM image of the flakes (Figure 4a), we can see that the PCLC flakes can copy the shape of the PDMS molds exactly. We can also see that the PCLC alignment inside the flake is not perfect; there are some dark dots that represent domains with different alignment directions, especially near the edges. This is because there is no alignment layer in the PDMS mold, but the LCs tend to align parallel at the interface with PDMS and PVA. This is conflicting with the rotational tendency of the cholesteric liquid crystals at the edges, so the alignment is not very uniform. When the size of flakes becomes larger, the influence of the edges becomes smaller and the alignment is better. In order to learn more about the morphology of the PCLC flakes, we used a 3D profilometer to observe the topography of the PCLC flakes. From Figure $4 \mathrm{~b}$, we can see that the major part of the PCLC flakes surface is flat, but there are a few irregular bumps. Figure $4 \mathrm{c}$,d shows a PCLC flake adhering on the glass plate when we polymerized the PCLC flake without taking off the glass plate. It can be seen that there are some concentric circles on the top of the flake; this means the bottom of the PDMS mold is not entirely flat. However, as the height of the concentric circles is relatively low, it will not influence the performance of the flakes. 


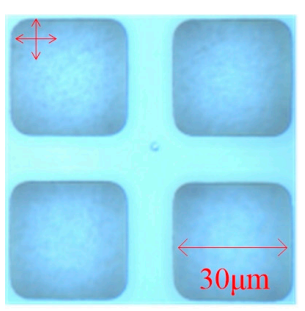

(a)

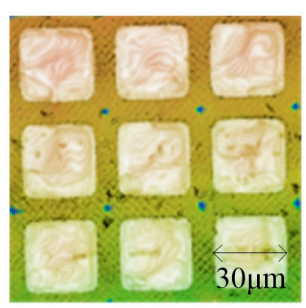

(b)

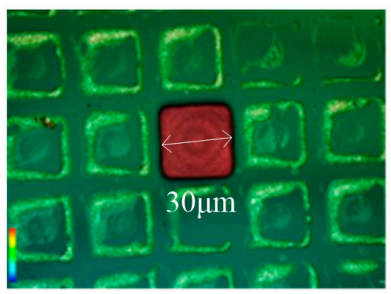

(c)

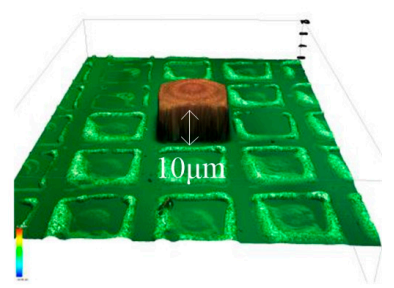

(d)

Figure 4. Characterization of flakes: (a) POM image of PCLC flakes in a PDMS mold; (b) The topography of PCLC flakes in PDMS mold; (c) the width of a single PCLC flake; (d) the height of a single PCLC flake.

The reflectance of PCLC flakes with different aspect ratios (length/width) inside a PDMS mold is shown in Figure 5a. The aspect ratios of PCLC flakes are 1:1, 2:1, and 3:1 and the sizes of the flakes are $60 \mu \mathrm{m} \times 20 \mu \mathrm{m}, 60 \mu \mathrm{m} \times 30 \mu \mathrm{m}$, and $60 \mu \mathrm{m} \times 60 \mu \mathrm{m}$, respectively. From Figure 5a, we can find that the reflectance of PCLC flakes at $760 \mathrm{~nm}$ wavelength is $15 \%, 22 \%$, and $28 \%$, respectively. The reflectance cannot reach $50 \%$ because the PCLC flakes only occupy a part of the whole area and they are too small to measure solely. More specifically, the size of light beam of the measurement instrument is much larger compared to the PCLC flakes. From Figure $5 b-d$, we can find that each size of PCLC flake occupies a different proportion of the whole area. The flake with a 3:1 aspect ratio occupies $37.5 \%$, the flake with a $2: 1$ aspect ratio occupies $45 \%$ and the flake with a $1: 1$ aspect ratio occupies $56.25 \%$. Since the reflection peak is only caused by the reflection of PCLC flakes and the PDMS mold has no contribution to it, the reflectance of the flakes can be calculated by the reflectance of PCLC flakes in a PDMS mold divided by the occupied area. Thus, the real transmittances of the PCLC flakes are $40 \%$, $48.9 \%$ and $49.8 \%$, respectively. So, the reflectance of larger PCLC flakes can reach the maximum value, which means the alignment inside the flakes is quite good. However, when the flake size is small, the alignment is influenced by the PDMS mold walls and the reflectance is lower. What is more, the irregular transmittance in the $600-700 \mathrm{~nm}$ wavelength range of Figure $5 \mathrm{a}$ is caused by the scattering of the PDMS mold.

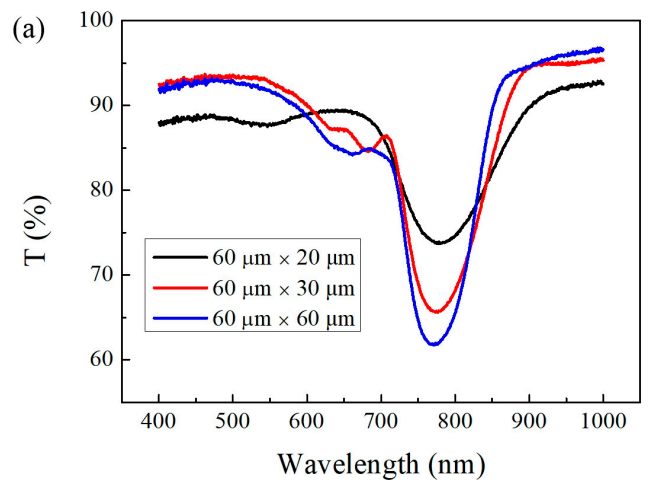

(b)

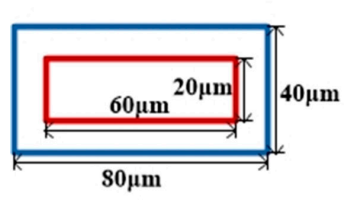

(c)

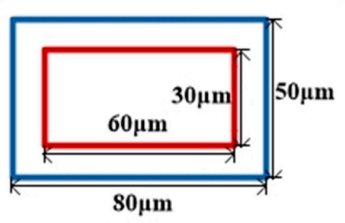

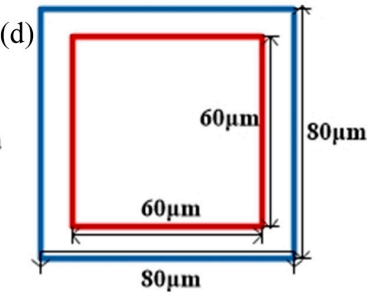

Figure 5. The transmittance and diagram of PCLC flakes in PDMS molds: (a) Transmittance of PCLC flakes in PDMS molds; (b) the diagram of the $60 \mu \mathrm{m} \times 20 \mu \mathrm{m}$ size; (c) The diagram of the $60 \mu \mathrm{m} \times 30 \mu \mathrm{m}$ size; (d) the diagram of the $60 \mu \mathrm{m} \times 60 \mu \mathrm{m}$ size (The blue line means the PDMS mold and the red line means the PCLC flakes). 


\subsection{Influence of PVA Layer Thickness}

PVA is a water-soluble synthetic polymer. It is widely used in paper-making, textiles, and a variety of coatings [11]. It can also provide a parallel orientation of liquid crystals. Herein, in order to release the PCLC flakes from the PDMS mold more conveniently and keep the parallel orientation of the liquid crystals, we spin-coated a layer of PVA over the PDMS mold. Because different speeds of spin-coating will influence the thickness of the PVA layer and the depth of the PDMS mold, we used four spin-coating speeds to get different depths of the PDMS mold, including $500 \mathrm{rpm}, 1000 \mathrm{rpm}$, $1500 \mathrm{rpm}$, and $2000 \mathrm{rpm}$. The spin-coat time was $30 \mathrm{~s}$. We used the $60 \mu \mathrm{m} \times 60 \mu \mathrm{m}$ PDMS mold to study the influence of the PVA layer thickness.

The depth of the PDMS mold after coating the PVA layer at different speeds can be seen from Figure 6a. We found that the PVA is mainly located at the bottom of the molds rather than at the walls. With the speed of spin-coating increasing, the PDMS mold becomes deeper because the PVA layer becomes thinner. The thickness of the PVA layer $h_{f}$ can be calculated by Equation (1):

$$
h_{f}=\left(\frac{3}{2}\right)^{\frac{1}{3}} a_{0}\left(1-a_{0}\right)^{-\frac{1}{3}} \omega^{-\frac{2}{3}} v_{0}^{\frac{1}{3}} e^{\frac{1}{3}}
$$

where $a_{0}$ is the solute concentration of the PVA solution, $\omega$ is the angular speed, $v_{0}$ is the initial viscosity of the fluid, and $e$ is the volatilization rate of the fluid [12-15]. Therefore, with the speed of spin-coating increasing, the $h_{f}$ of the PVA layer becomes thinner. Accordingly, the depth of the PDMS mold becomes deeper and the PCLC flakes become thicker.
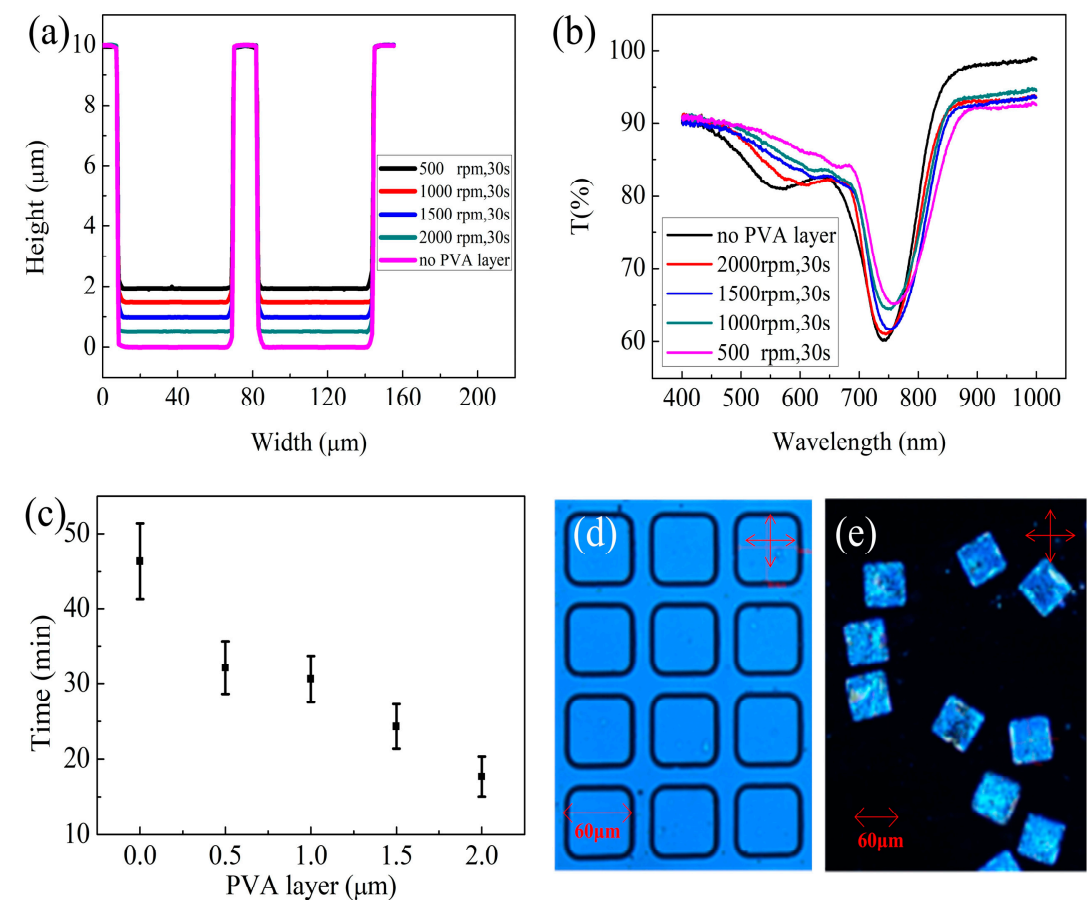

Figure 6. Influence of the PVA layer thickness on the reflectance and release time of the PCLC flakes from the $60 \mu \mathrm{m} \times 60 \mu \mathrm{m}$ size PDMS mold: (a) Surface profiles of the PDMS mold after spin-coating PVA at different speeds; (b) transmittance of PCLC flakes with different thicknesses; (c) The release times of different thicknesses of PVA layers; (d) the POM image of the PDMS mold after flakes were released; (e) the POM image of the released PCLC flakes.

Furthermore, in order to study the influence of the PVA layer on the reflectance of the PCLC flakes, we tested the transmittance of the PCLC flakes in the PDMS mold with five different PVA thicknesses, as shown in Figure $6 \mathrm{~b}$. We can see that the reflectance of the PCLC flakes becomes lower when the 
spin-coating speed becomes lower, which means the PVA layer becomes thicker and the thickness of flakes becomes thinner. The reflectance $R$ of a PCLC layer can be calculated by Equation (2) [16]:

$$
\begin{gathered}
R=1-\exp \left(-\frac{2 \pi \sigma \bar{n} d}{\lambda_{0}}\right) \\
\text { where, } \sigma=\left[\sqrt{4\left(\frac{\lambda_{0}}{\bar{n} \cdot P}\right)^{2}+\left(\frac{n_{e}^{2}-n_{o}^{2}}{n_{e}^{2}+n_{o}^{2}}\right)^{2}}-1-\left(\frac{\lambda_{0}}{\bar{n} \cdot P}\right)^{2}\right]^{\frac{1}{2}} \\
\bar{n}=\sqrt{\frac{n_{e}^{2}+n_{o}^{2}}{2}}
\end{gathered}
$$

From the Equation (2) we can see that when the thickness $d$ decreases, the reflectance also will decreases. With the thickest PVA layer, the reflectance of PCLC flakes in the PDMS mold is $24 \%$; since the PCLC flakes only occupy $56.25 \%$ of the area in the PDMS molds, the reflectance of PCLC flakes is about $43.7 \%$. When the PVA layer is thinner than $1 \mu \mathrm{m}$, the influence of the PVA layer on the reflectance of the PCLC flakes is very small, less than $2 \%$. Therefore, the PVA layer influences the reflectance of the PCLC flakes because the flakes become thinner, but the influence is very small when the PVA layer is thinner than $1 \mu \mathrm{m}$.

The release time of the PCLC flakes in the PDMS mold with different PVA thicknesses is shown in Figure 6c. From this figure we can see that the release time of the flakes from a mold without a PVA layer is almost $50 \mathrm{~min}$. Sometimes, there are still some flakes remaining inside the mold without a PVA after $1 \mathrm{~h}$ of ultrasonic vibration and the mold may be damaged. The releasing process is very time consuming and the PDMS mold could hardly be used again when a PVA layer was not used. After coating a PVA layer inside the molds, the release time drops significantly to less than $30 \mathrm{~min}$ and the release time becomes shorter when the PVA layer becomes thicker. When the PVA layer is $2 \mu \mathrm{m}$, the release time is only about $17 \mathrm{~min}$. The PDMS mold (with $1 \mu \mathrm{m}$ thick PVA layer) after release of the PCLC flakes is shown in Figure 6d. It shows that the PDMS molds can maintain their original shape and are unbroken. Since PVA can be dissolved in water, the mold can be reused after cleaning. The released PCLC flakes are shown in Figure 6e. The flakes are neat and uniform which is an improvement over the previous result. So coating a layer of PVA in the PDMS molds for PCLC flake fabrication using the soft lithography method can make the mold recyclable and save time.

\subsection{Influence of Mold Shape}

For different applications, the requirements for the PCLC flake size are also different. For example, the size of PCLC flakes influences the electric driving property of an electricity responsive device [8]. Therefore, we also studied the influence of PDMS mold shape. Three different mold shapes $(60 \mu \mathrm{m} \times 60 \mu \mathrm{m}, 60 \mu \mathrm{m} \times 30 \mu \mathrm{m}$ and $60 \mu \mathrm{m} \times 20 \mu \mathrm{m})$ were selected to study the influence of mold shape. The surface profiles of the PDMS molds with different shapes after spin-coating PVA at different speeds are shown in Figure 6a, Figure 7a, and Figure 7b. From the results, we can see that the PVA layer thickness is not be influenced by the mold shape and is only influenced by the spin-coating speed. The release time of the PCLC flakes in the PDMS molds with different shapes are shown in Figure 6b, Figure 7c, and Figure 7d. From the result, we can see that in all kinds of mold, the release time is shorter when the PVA layer is thicker. At the same time, the release time is also shorter when the mold is narrower and smaller. For flakes made using a $60 \mu \mathrm{m} \times 20 \mu \mathrm{m}$ size mold with a $2 \mu \mathrm{m}$ PVA layer, the release time is less than $15 \mathrm{~min}$. However, the influence of the mold shape is not very significant; the difference between release times with different molds is only $30 \%$, while the difference in release time with different PVA layer thicknesses can reach $67 \%$. 

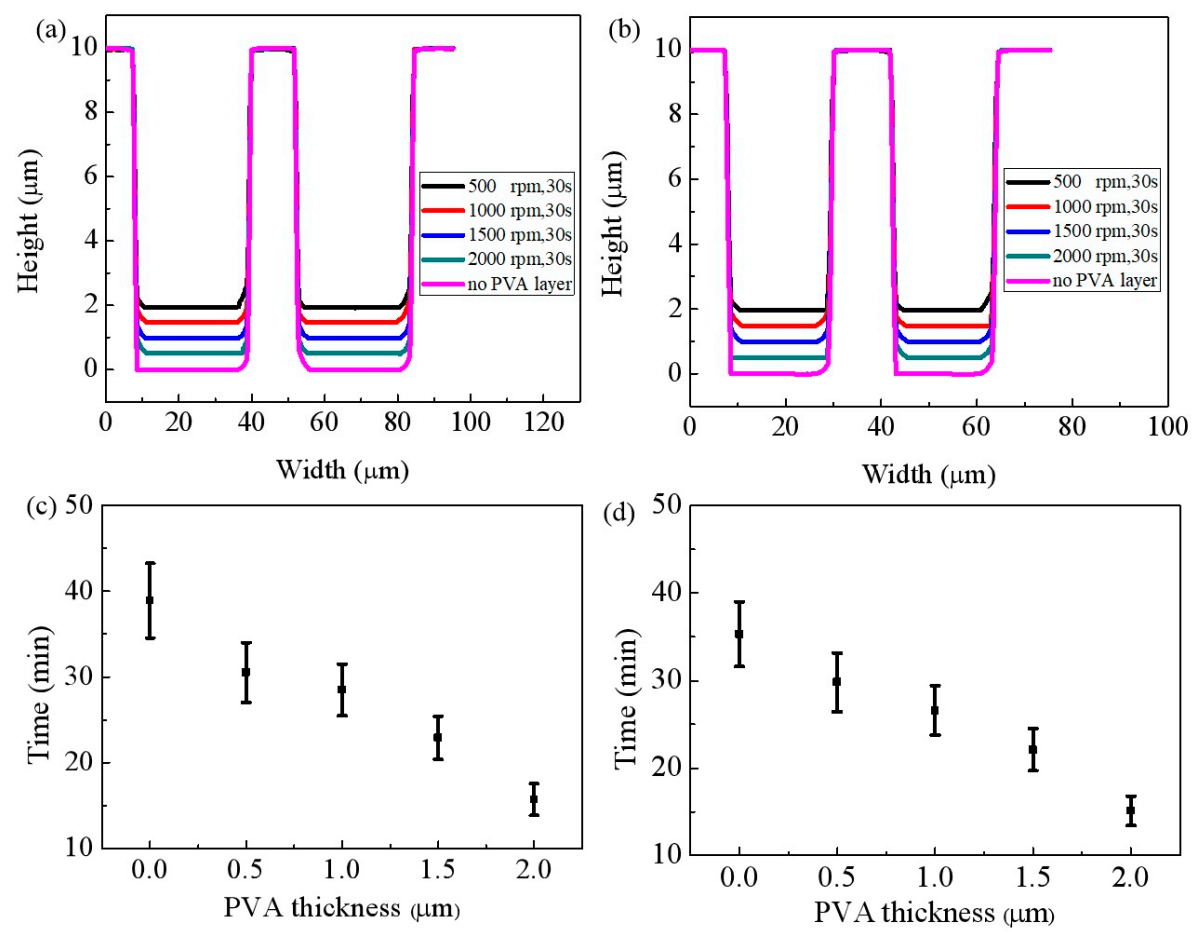

Figure 7. Influence of different mold shapes: (a,b) Surface profiles of $60 \mu \mathrm{m} \times 30 \mu \mathrm{m}$ size and $60 \mu \mathrm{m} \times 20 \mu \mathrm{m}$ size PDMS molds after spin-coating PVA at different speeds; (c,d) the release time of PCLC flakes in $60 \mu \mathrm{m} \times 30 \mu \mathrm{m}$ size and $60 \mu \mathrm{m} \times 20 \mu \mathrm{m}$ size PDMS molds with different PVA layer thicknesses.

\section{Conclusions}

Since each PCLC flake can selectively reflect light, it holds promise as an alternative display technology in flexible, reflective displays and other applications such as tunable infrared reflectors. In this paper, we have demonstrated a simple technique for manufacturing shaped PCLC flakes of different sizes using soft lithography. A flexible PDMS mold was made as an inverse replica of silicon wafers patterned with different features. In order to release the PCLC flakes without breaking them and to be able to reuse the PDMS mold, a PVA layer was coated upon the PDMS mold which helped to release the PCLC flakes easier and faster. The alignment and morphology of PCLC flakes made by this method is quite good and the reflectance of the flakes of full size can reach $50 \%$. The thickness of the PVA layer will influence the reflectance and release time of the PCLC flakes. When the PVA layer is thicker, the reflectance of the PCLC flakes is lower and the release time is shorter. The shape of the PDMS mold can also influence the release time of PCLC flakes, but not significantly.

Supplementary Materials: The following are available online at http://www.mdpi.com/2072-666X/10/7/441/s1, Figure S1: DSC result of the monomer mixture.

Author Contributions: Conceptualization, G.Z. (Guanqing Zhou) and D.Y.; data curation, G.Z. (Guanqing Zhou) and S.L.; methodology, G.Z. (Guanqing Zhou) and W.L.; writing-original draft preparation, G.Z. (Guanqing Zhou) and D.Y.; writing—review and editing, G.Z. (Guofu Zhou); and supervision, D.Y. and G.Z. (Guofu Zhou).

Funding: This research was funded by the National Natural Science Foundation of China (Nos. 21711530647 and 51561135014), Science and technology project of Guangdong Province (Nos. 2018A050501012 and 2017B020240002).

Acknowledgments: This work was also supported by the National Center for International Research on Green Optoelectronics, MOE International Laboratory for Optical Information Technologies, Guangdong Provincial Key Laboratory of Optical Information Materials and Technology, the 111 Project and Yunnan expert workstation (No. 2017IC011).

Conflicts of Interest: The authors declare no conflict of interest. The funders had no role in the design of the study; in the collection, analyses, or interpretation of data; in the writing of the manuscript, or in the decision to publish the results. 


\section{References}

1. Liu, D.; Bastiaansen, C.W.; Toonder, J.M.; Broer, D.J. Photo-switchable surface topologies in chiral nematic coatings. Angew. Chem. Int. Ed. 2012, 51, 892-896. [CrossRef] [PubMed]

2. Khandelwal, H.; Loonen, R.G.; Hensen, J.M.; Schenning, A.H.; Debije, M.G. Application of broadband infrared reflector based on cholesteric liquid crystal polymer bilayer film to windows and its impact on reducing the energy consumption in buildings. J. Mater. Chem. A 2014, 2, 14622-14627. [CrossRef]

3. Khandelwal, H.; Loonen, R.G.; Hensen, J.M.; Debije, M.G.; Schenning, A.H. Electrically switchable polymer stabilised broadband infrared reflectors and their potential as smart windows for energy saving in buildings. Sci. Rep. 2015, 5, 11773. [CrossRef] [PubMed]

4. Kendhale, A.M.; Schenning, A.P.H.J.; Debije, M.G. Superior alignment of multi-chromophoric perylenebisimides in nematic liquid crystals and their application in switchable optical waveguides. J. Mater. Chem. A 2012, 1, 229-232. [CrossRef]

5. Zhou, G.; Yuan, D.; Liu, Y.; Lin, X.; Zhou, G.; Li, N. Properties of Liquid Crystal Polymer Films for Electricity-responsive IR Reflective Windows. Acta Photonica Sin. 2017, 46, 0331002. [CrossRef]

6. Korenic, E.M.; Jacobs, S.D.; Fare, S.M.; Li, L. Cholesteric Liquid Crystal Flakes-A New Form of Domain. Mol. Crysr. Liq. Cryst. 1998, 317, 197-219. [CrossRef]

7. Oudshoorn, M.H.M.; Penterman, R.; Rissmann, R.; Bouwstra, J.A.; Broer, D.J.; Hennink, W.E. Preparation and characterization of structured hydrogel microparticles based on cross-linked hyperbranched polyglycerol. Langmuir 2007, 23, 11819-11825. [CrossRef] [PubMed]

8. Kosc, T.Z.; Marshall, K.L.; Trajkovska-Petkoska, A.; Kimball, E.; Jacobs, S.D. Progress in the development of polymer cholesteric liquid crystal flakes for display applications. Displays 2004, 25, 171-176. [CrossRef]

9. Trajkovska-Petkoska, A.; Varshneya, R.; Kosc, T.Z.; Marshall, K.L.; Jacobs, S.D. Enhanced Electro-Optic Behavior for Shaped Polymer Cholesteric Liquid-Crystal Flakes Made Using Soft Lithography. Adv. Funct. Mater. 2010, 15, 217-222. [CrossRef]

10. Petkoska, A.T.; Jacobs, S.D. The Manufacture, Characterization and Manipulation of Polymer Cholesteric Liquid Crystal Flakes and Their Possible Applications. J. Mater. Sci. Eng. A 2012, 2, 137-151.

11. Demerlis, C.C.; Schoneker, D.R. Review of the oral toxicity of polyvinyl alcohol (PVA). Food Chem. Toxicol. 2003, 41, 319-326. [CrossRef]

12. Lawrence, C.J. The mechanics of spin coating of polymer films. Phys. Fluids 1988, 31, 2786-2795. [CrossRef]

13. Jenekhe, S.A. Effects of solvent mass transfer on flow of polymer solutions on a flat rotating disk. Ind. Eng. Chem. Fundam. 1984, 23, 425-432. [CrossRef]

14. Washo, B.D. Rheology and Modeling of the Spin Coating Process. IBM J. Res. Dev. 1977, 21, $190-198$. [CrossRef]

15. Meyerhofer, D. Characteristics of resist films produced by spinning. J. Appl. Phys. 1978, 49, 3993. [CrossRef]

16. Belyakov, V.A. Diffraction Optics of Complex-Structured Periodic Media: Localized Optical Modes of Spiral Media; Springer: New York, NY, USA, 2019.

(C) 2019 by the authors. Licensee MDPI, Basel, Switzerland. This article is an open access article distributed under the terms and conditions of the Creative Commons Attribution (CC BY) license (http://creativecommons.org/licenses/by/4.0/). 